\title{
Integration of Space and In Situ Observations to Study Global Climate Change
}

Abstract

The currently available model-based global data sets of atmospheric circulation are a by-product of the daily requirement of producing initial conditions for numerical weather prediction (NWP) models. These data sets have been quite useful for studying fundamental dynamical and physical processes, and for describing the nature of the general circulation of the atmosphere. However, due to limitations in the early data assimilation systems and inconsistencies caused by numerous model changes, the available model-based global data sets may not be suitable for studying global climate change.

A comprehensive analysis of global observations based on a fourdimensional data assimilation system with a realistic physical model should be undertaken to integrate space and in situ observations to produce internally consistent, homogeneous, multivariate data sets for the earth's climate system. The concept is equally applicable for producing data sets for the atmosphere, the oceans, and the biosphere, and such data sets will be quite useful for studying global climate change.

\section{Introduction}

The last ten years have seen a rapid development in atmospheric modeling and the beginning of operational numerical weather prediction (NWP) for the whole globe. This development has been possible due to advances in the understanding of atmospheric dynamics and the very rapid advances in computer technology which, together with more economical integration methods, have made it possible to significantly increase horizontal and vertical resolution and hence make more accurate calculations of dynamical and physical processes. Today, the European Centre for Medium Range Weather Forecasting (ECMWF), for instance, produces daily global forecasts of up to ten days using a spectral model that has 106 wavenumbers in triangular truncation and 19 vertical levels.

Over the same period, data assimilation methods have gradually developed to make possible the use of unconventional and nonsynoptic observations from satellites, drifting buoys, and aircraft. Although only minor changes in the global observing system have taken place since the time of the Global Weather Experiment (also referred to as the First GARP [Global Atmospheric Research Program] Experiment, FGGE) in 1979 (see Table 1) the accuracy of short-range forecasts has improved significantly (see Figure 1). According to Lange and Hellsten

\footnotetext{
${ }^{1}$ European Centre for Medium Range Weather Forecasts, Shinfield Park, Reading, Berkshire RG2 9AX, England.

${ }^{2}$ Center for Ocean-Land-Atmosphere Interactions, Department of Meteorology, University of Maryland, College Park, MD, 20742.
}

(C) 1988 American Meteorological Society
(1986), the three-day root mean square (RMS) forecast error (12-month running average) for the Northern Hemisphere (NH) between 1979 and 1986 has been reduced by more than 35 percent. During the same period, useful predictive skill has been extended from between three and four days to about seven days for NH (Bengtsson, 1985). The main improvement has taken place at middle and high latitudes of $\mathrm{NH}$; in the tropics improvements have been less significant, due to lack of appropriate observations and deficiencies in the formulation of the relevant physical processes.

As discussed by Lorenz (1982), there is further scope for improvement in predictive skill by improving models. Figure 2, taken in part from Lorenz (1982), shows the potential improvement in predictive skill by comparing the error growth of the ECMWF model for the winter of 1980-1981 with the error growth of an assumed perfect model (dashed curve). Error growth of the perfect model has been obtained by comparing the RMS 500-mb height differences of 100 ten-day consecutive operational forecasts separated by one day. This second 19801981 dashed curve at day one is the RMS 500-mb height difference between a one-day forecast and observed state of the atmosphere, and gives a measure of predictability for an initial perturbation that is equal to the error of a one-day forecast. (For further information see Lorenz, 1982, and Hollingsworth et al., 1987). Figure 2 also shows a similar calculation for the winter of 1985-1986. The one-day forecast error has been reduced compared to the winter of 1980-1981, due to improvements in both the model and the data assimilation system that have taken place over the intervening period. The ECMWF model's error growth rate, which is an estimate of predictability, is also lower. The explanation for this improvement is not straightforward, but is, in all likelihood, due to improvements in the forecast model and the data assimilation that have led to more accurate specifications of the initial states and more consistent analyses.

During the last ten years the ECMWF data assimilation system has undergone significant improvements, and the total short range forecast errors have been reduced by more than one half, demonstrating that present data assimilation systems are superior to those used in the past in integrating observations irregularily distributed in time and space and obtained by quite different observational technologies. Derived quantities, such as the diabatic-heating fields and the surface heat fluxes, are particularly affected by the parameterization of physical processes (Simonot and LeTreut, 1987). The changes in the data assimilation have taken place step by step as model analysis and initialization improvements have been gradually introduced into the operational system. In data sparse regions, such as parts of the tropics and the Southern Hemisphere $(\mathrm{SH})$, the analyzed fields and, in particular, derived quantities are affected 
TABLE 1. Number of reports (obs.) received at ECMWF at $1200 \mathrm{UTC} \pm 3$ hours.

\begin{tabular}{|c|c|c|c|c|c|c|c|}
\hline \multirow[b]{2}{*}{ Obs. Type } & \multicolumn{7}{|c|}{ Year } \\
\hline & 1981 & 1982 & 1983 & 1984 & 1985 & 1986 & 1987 (Feb) \\
\hline SYNOP & 6000 & 6000 & 6000 & 6000 & 6000 & 6000 & 6500 \\
\hline SHIP & 800 & 900 & 1000 & 1050 & 1050 & 1000 & 1100 \\
\hline $\begin{array}{l}\text { TEMP } \\
\text { (Part A) }\end{array}$ & 600 & 600 & 600 & 600 & 600 & 610 & 630 \\
\hline $\begin{array}{l}\text { PILOT } \\
\text { (Part A) }\end{array}$ & 200 & 210 & 210 & 210 & 220 & 230 & 200 \\
\hline AIREP & 350 & 450 & 550 & 750 & 800 & 900 & 820 \\
\hline SATEM & 900 & 900 & 900 & 900 & - & - & - \\
\hline TOVS & - & - & - & - & 2800 & 4600 & 2000 \\
\hline SATOB & 600 & 1000 & 1200 & 1200 & 1300 & 1300 & 1350 \\
\hline DRIBU & 120 & 120 & 120 & 130 & 200 & 500 & 550 \\
\hline
\end{tabular}

Note: Number of global observations received at ECMWF in a 6-hour interval; 1200 UTC \pm 3 hours. The number of observing platforms is less; for SYNOP around 3500 and DRIBU's (drifting buoys) 178 during 1987. The reference period for TOVS during 1986 includes one polar orbiting satellite only.

\section{ECMWF FORECAST SKILL January 1980 - January 1988}

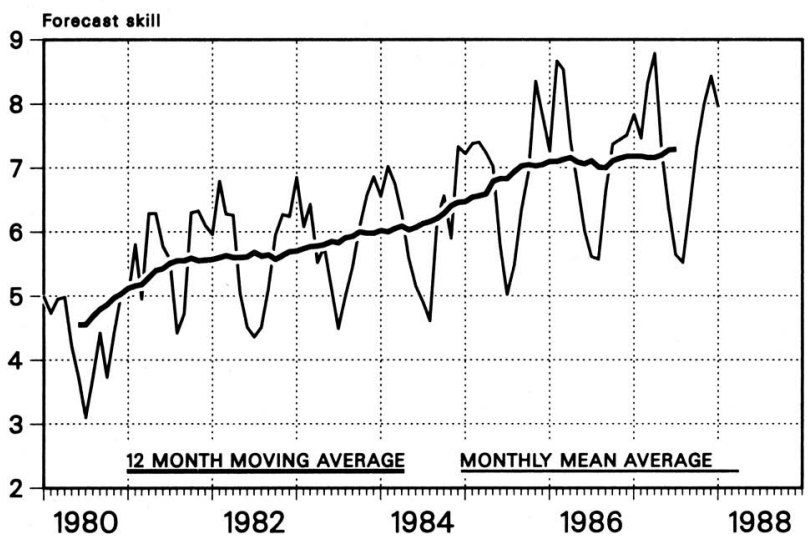

FIG. 1. ECMWF Northern Hemisphere forecast skill from January 1980 to January 1988 for geopotential thickness (1000 to $200 \mathrm{mb})$ and average temperature forecasts $(850$ to $200 \mathrm{mb}$ ). (See Appendix 1). Thin line, monthly mean values; thick line, 12-month running means.

by the analysis-forecast system.

As a result of this progress we are reaching a stage at which the information available in the first guess of the model, prior to the analysis process, for height and wind over data dense regions such as for Europe and the United States, is comparable to the information from current individual observations (Hollingsworth et al., 1986). Comprehensive analysis of global atmospheric fields, based on operational four-dimensional assimilation of all available upper-air and surface observations constitutes the most reliable observational basis for studying and comprehending fundamental dynamical and physical processes in the atmosphere.

The limitations of the early data assimilation systems and inconsistencies caused by numerous model changes make it necessary to consider whether there might be reason to undertake a reanalysis of past data using the best available data assimilation system. It is argued that such an effort is well-justified and should be given high priority within the context of the World Climate Research Program and the interdisciplinary program of earth-system science and global climate change.
In Section 2, the limitations of the currently available modelbased data sets as produced by the frequently changing analysis-forecast systems at the operational (NWP) centers are summarized. Section 3 describes the global data problem and highlights the role of advanced models in filling in some of the gaps in the observations. In Section 4 it is shown that, compared to the earlier analyses, the structure and the energetics of the global atmosphere for the Global Weather Experiment year 1979 is significantly different if the currently available advanced global model and assimilation system is used to analyze the same observations. Based on the greater internal consistency and the improved forecasts, it is also argued that the revised analyses are clearly superior to the earlier analyses. Some important diagnostic studies that could be performed if an internally consistent, homogeneous, multivariate time series of atmospheric variables were produced by reanalyzing the past data sets are listed in Section 6. Section 6 also gives the concluding remarks in which it is pointed out that the concept of providing reanalyzed data sets would be equally applicable for defining the four-dimensional structure of the ocean's circulation, and particularly those in the tropical oceans, which can be forced by improved fields of reanalyzed atmospheric forcing functions.

\section{Limitations of the currently available model-based data sets}

The currently available model-based data sets were primarily produced by the operational NWP centers as a byproduct of the daily requirement of producing initial conditions for the NWP models. Northern hemispheric analyses of the twice daily circulation fields produced by the National Meteorological Center (NMC) of the United States for the period 1963 to 1978 and global analyses produced both by NMC and ECMWF, for the period 1978 to the present are but a few examples of such data sets. Quite a large number of useful and insightful diagnostic studies of the general circulation of the atmosphere have been carried out utilizing these data sets. However, these data sets do have several deficiencies (Trenberth and Olson, 1988a, 1988b), some of which are mentioned below.

\section{a. Frequent changes in the forecast models}

Operational NWP was started only about 35 years ago and the 


\section{LORENZ CURVES}

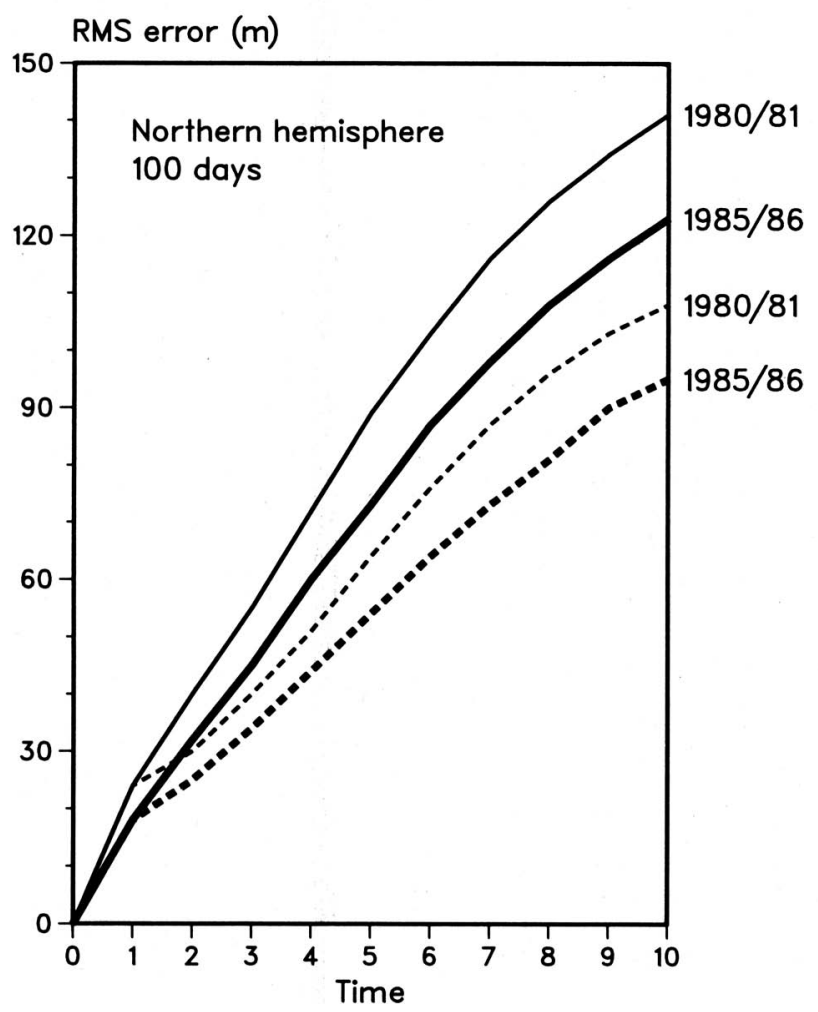

FIG. 2. Predicted skill of the ECMWF model solid for winter 1980/ 81, and winter 1985/86 (full line). Dashed line gives an estimate of predictability (Lorenz 1982) for each of the winters. (After Hollingsworth et al., 1985, with the permission of the Royal Meteorological Society.)

models were extremely simple (viz. barotropic or quasi-geostrophic, etc.). Multilevel primitive equation models have been used for operational NWP for less than 25 years. During this evolutionary phase of NWP, models have been continuously improved to reduce forecast errors due to deficiencies in the treatment of various aspects of physical and numerical processes. This improvement is still continuing. Table 2 shows as an example the majer changes in the ECMWF forecast model over the last eight years. ${ }^{3}$

The existing data sets, in particular the earlier ones, are particularly inadequate for research required to support programs such as TOGA (Tropical Ocean Global Atmosphere) and WOCE (World Ocean Circulation Experiment) because of the need for accurate global fields of both heat flux and wind stress at the ocean surface. In the past, the operational NWP models did not have a realistic treatment of the planetary boundary layer. Development of forecast models has been traditionally motivated by the ability and limitations of the models in analyzing and predicting the synoptic and large-scale dynamical circulation, particularly in the extratropics. It is only recently that some attention is being paid to the importance of physical processes at the air-sea and air-land interfaces. In order to

\footnotetext{
${ }^{3}$ For a recent evaluation of global analyses from NMC and ECMWF see Trenberth and Olson (1988a, 1988b).
}

produce improved data sets of surface-wind stress and surfaceheat fluxes, it will be essential to further improve both the parameterization of boundary-layer processes and the assimilation of boundary-layer data.

\section{b. Frequent changes in the data-assimilation system}

Development of data-assimilation techniques, particularly in the combination of conventionally derived data sets and satellite data sets, has also been a continuously evolving discipline of research. Table 3 shows the major changes in the ECMWF data assimilation system over the past eight years. These changes have clearly improved the analyses and forecasts.

\section{c. Unavailability of delayed mode data}

The operational constraint of producing a forecast every day by a certain time of the day makes it necessary to impose a cut-off time for inclusion of the incoming observations. Any data that are received after the cut-off time can not be utilized by the data-assimilation system. This operational constraint can cause data gaps in those areas from which data transmission

TABLE 2. Major changes in the ECMWF Forecast ModelSeptember 1979 to April 1987.

\begin{tabular}{|c|c|}
\hline & Model Change \\
\hline Date & $\begin{array}{l}\text { Original model implemented-September } 1979 \\
\text { N48 grid point model, } 15 \text { vertical levels, } \sigma \text { - } \\
\text { coordinates }\end{array}$ \\
\hline 27 July 1982 & SST climatology replaced by analysed SST \\
\hline 21 April 1983 & $\begin{array}{l}\text { Spectral model-T63, } 16 \text { vertical levels, } \\
\text { hybrid vertical coordinates, envelope } \\
\text { orography }\end{array}$ \\
\hline 2 May 1984 & Diurnal radiation cycle \\
\hline 4 December 1984 & $\begin{array}{l}\text { New parameterization of radiative transfer, } \\
\text { incorporation of stratospheric drag }\end{array}$ \\
\hline 1 May 1985 & $\begin{array}{l}\text { Spectral model-T106, incorporation of } \\
\text { shallow convection, modification of the Kuo } \\
\text { convective scheme, new interactive cloud } \\
\text { parameterization (incorporation of boundary } \\
\text { layer clouds) }\end{array}$ \\
\hline 13 May 1986 & $\begin{array}{l}19 \text { vertical levels- }-3 \text { additional stratospheric } \\
\text { levels }\end{array}$ \\
\hline 10 July 1986 & Gravity wave drag \\
\hline 7 April 1987 & $\begin{array}{l}\text { New parameterization of land surface processes } \\
\text { and vegetation }\end{array}$ \\
\hline
\end{tabular}

TABLE 3. Major changes in the ECMWF Data Assimilation System

\section{Data-Assimilation Changes}

Date

21 September 1982 22 May 1984

26 February 1985

4 March 1986

11 March 1986

9 September 1986
Operational implementation of first systemSeptember 1979

Multivariate $\mathrm{O} / \mathrm{I}$ analysis-nonlinear normal mode initialization

Diabatic initialization

Revised analysis scheme (refined structure functions)

Use of high-resolution satellite surroundings (TOVS, $250 \mathrm{~km}$ )

Special handling of tidal signals in initialization

Use of precipitable water derived from polar orbiting satellites

Analysis at model levels (hybrid coordinates), further refined structure functions 


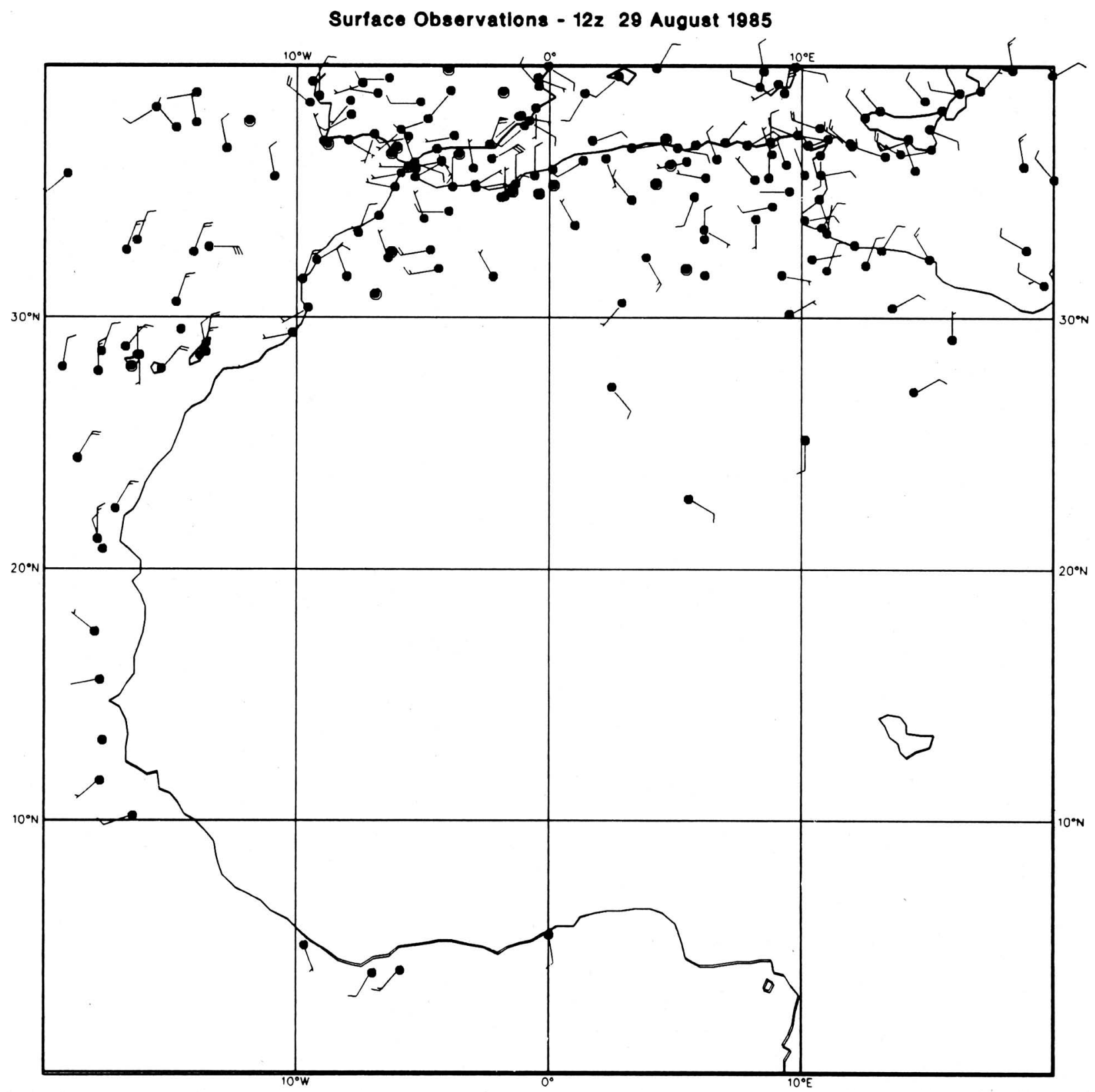

FIG. 3. Surface observations for 12 UTC, 29 August 1985.

or reception was delayed. Under a comprehensive plan for reanalyzing past data sets for TOGA, WOCE, and other programs under the auspices of earth-system science and global climate change, it would be possible to collect and archive delayed mode data sets so that they can also be assimilated along with the other observations.

It should be noted, however, that the requirements of producing daily analyses and forecasts necessitate an initial evaluation of the quality of all the data sets that are received at the NWP centers, which helps in detecting major sources of error that may be either instrumental or due to problems of transmission or due to errors in the algorithms for deriving satellite soundings. It is therefore essential that every effort should be made to make all the data sets available in real time to the extent that it is possible. It would also be desirable to archive these data sets along with a documentation of operational problems.

\section{The Global Data Problem}

Studies of atmospheric processes of significance for both climate research and medium- and extended-range prediction require global observations, through the whole depth of the atmosphere, of the basic meteorological parameters: wind, temperature, and moisture. In addition, observations at ground level to define the surface boundary conditions are required. 


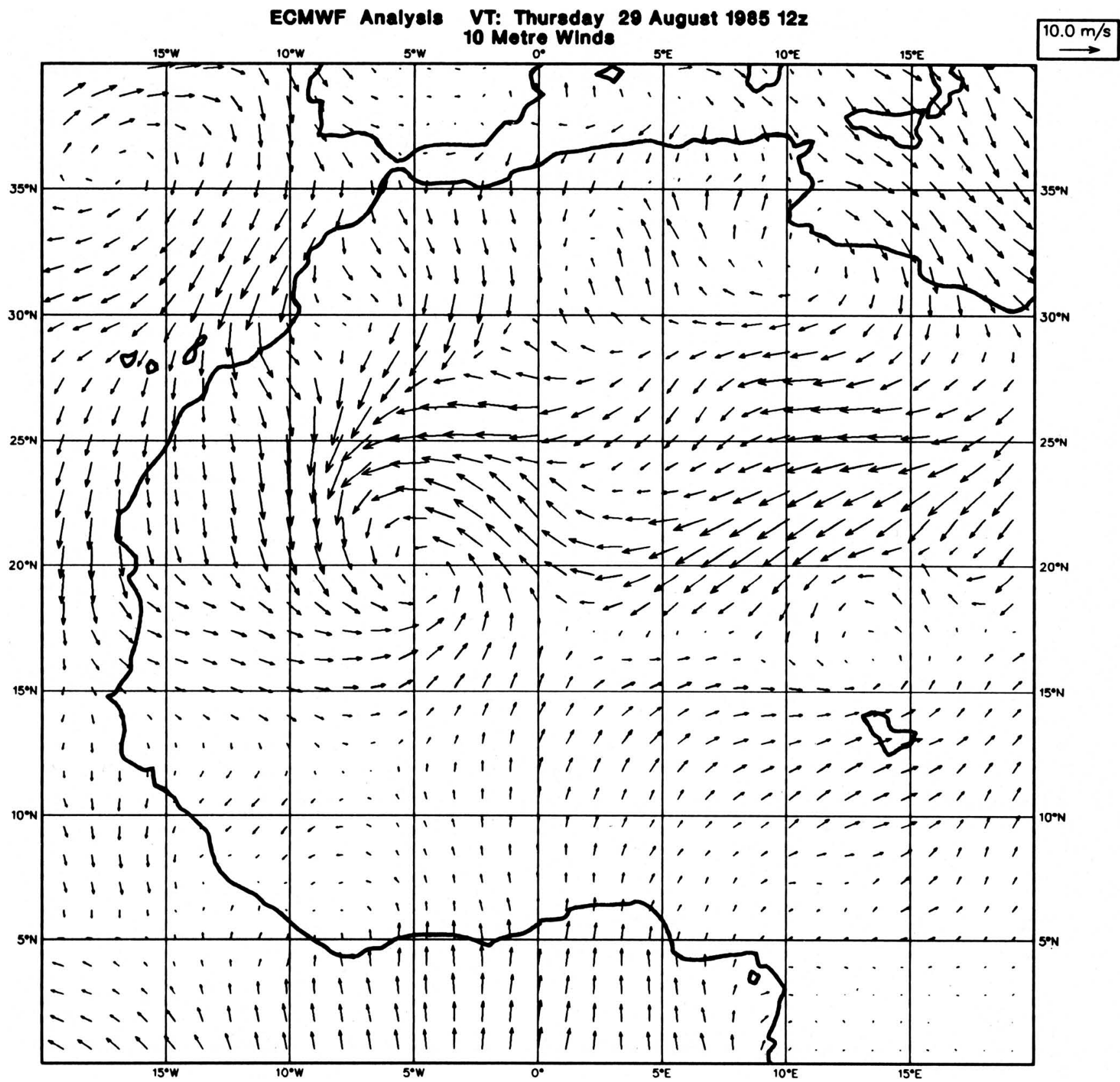

FIG. 4. ECMWF analysis for 12 UTC, 29 August 1985.

The space and time resolution of observations might be expected to be commensurate with the resolution of the atmospheric model (i.e., 50-100 km for models expected to become available over the next few years). Fortunately this daunting requirement can be relaxed due to the strong dynamical and physical coupling between meteorological observations in space and time. As first demonstrated by Smagorinsky et al., (1970), the kind of model being used 20 years ago was able to reconstitute the humidity field in most of its details after a day or two even if detailed humidity data were missing in the initial state. There also exists a large body of evidence that suggests that a realistic, high-resolution dynamical model can simulate the formation and growth of small-scale weather systems (smaller than that which can be resolved by observations) even if only the relatively large-scale circulation features are explicitly described by global observations (Anthes, 1972; Dell'Osso and Bengtsson, 1985; Tuleya, 1987). In this respect, a realistic global model can be viewed as a unique and independent observing system that can generate information at a scale finer than that of the conventional observing system. This is possible only because the models are able to predict the short-term evolution of atmospheric circulation reasonably well.

Numerical experiments (e.g., Hollingsworth et al., 1986; Arpe, et al., 1985) have shown that, for extratropical prediction, an accurate specification of the wind and mass field in the baroclinic zones is crucial to forecast quality, while a detailed analysis of the boundary layer appears to be less impor- 
Wodnesday 28 August $198512 z$ ECMWF Forecast it 24 VT: Wodnesday 28 August $198500 z$

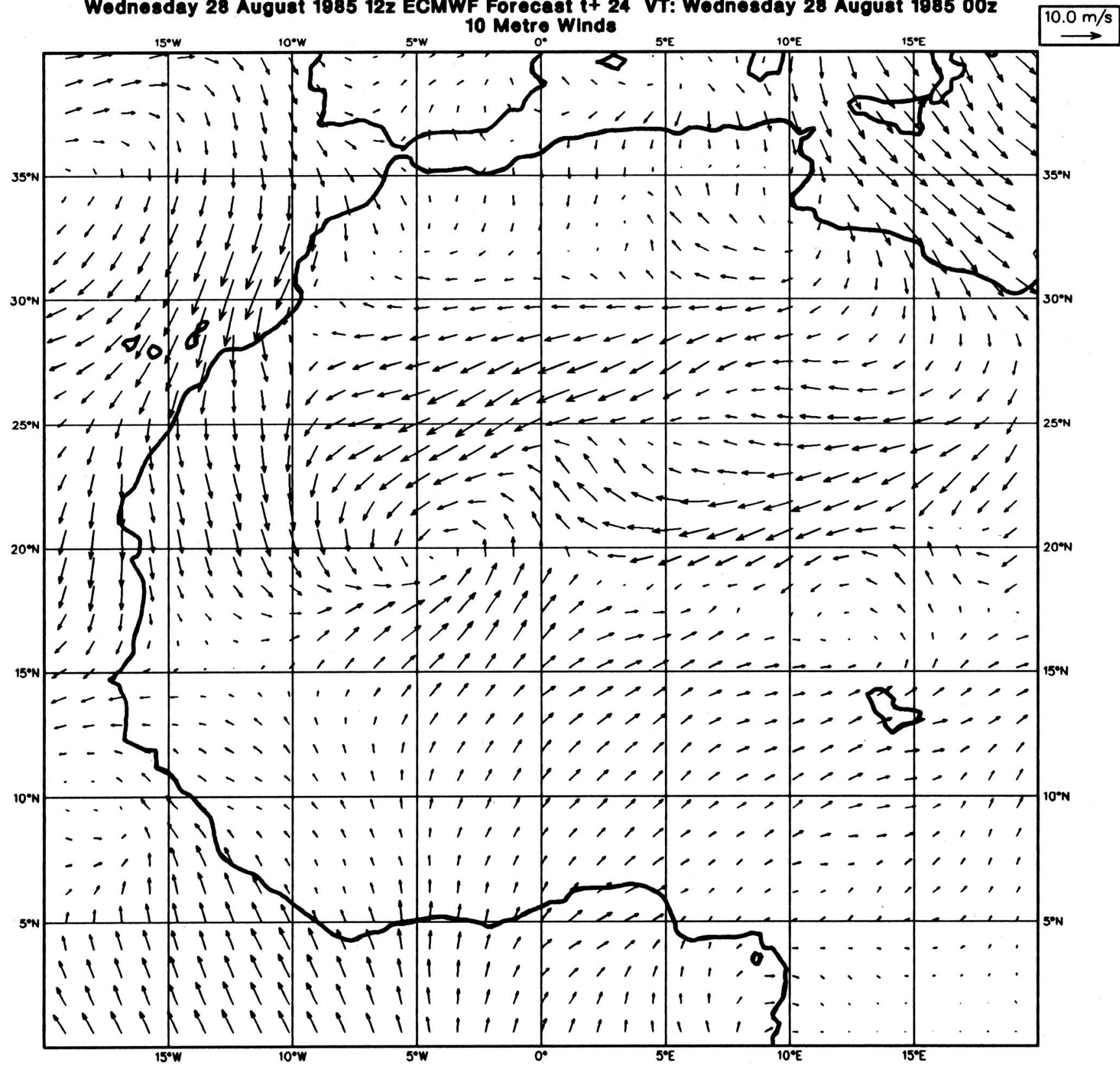

FIG. 5. Twenty-four hour forecast from initial condition of 12 UTC, 28 August 1985.

tant. The explanation again is that the models are able to generate some of the missing information, and it also indicates that there is some redundancy among the basic meteorological parameters in the atmosphere.

Figure 3 shows the surface observations at 12 UTC, 29 August 1985 over North Africa. Due to a technical problem at the regional telecommunication center in Paris during the period 25 to 30 August 1985, data were lost for the major part of North Africa. Figure 4 shows the wind field at $30 \mathrm{~m}$ above ground analyzed at ECMWF (the lowest level of the ECMWF model). As can be seen, an intense surface vortex has been analyzed with the center near $20^{\circ} \mathrm{N}, 5^{\circ} \mathrm{W}$. Since there are no available data whatsoever to analyze this feature of 12 UTC, 29 August (satellite temperature and wind data are not used over land) the vortex must have been present in the first guess field obtained by integrating the model. That this indeed was the case can be seen from Fig. 5, which shows the $24 \mathrm{hr}$ forecast from 12 UTC, 28 August 1985. A careful inspection of preceding forecasts shows that the vortex was generated near the Hoggar mountains, farther to the east, a few days earlier. The reality of the strong vortex is supported by the dust signature seen in the Meteosat infrared (IR) image for 1145 UTC, 29 August (Fig. 6). This example lends further support to the expectation that a good dynamical model is apparently capable of generating synoptic scale disturbances that form and amplify due to the dynamical instabilities of the large scale flow, if the latter can be described adequately by the observational network. For a more comprehensive discussion on this and other similar 


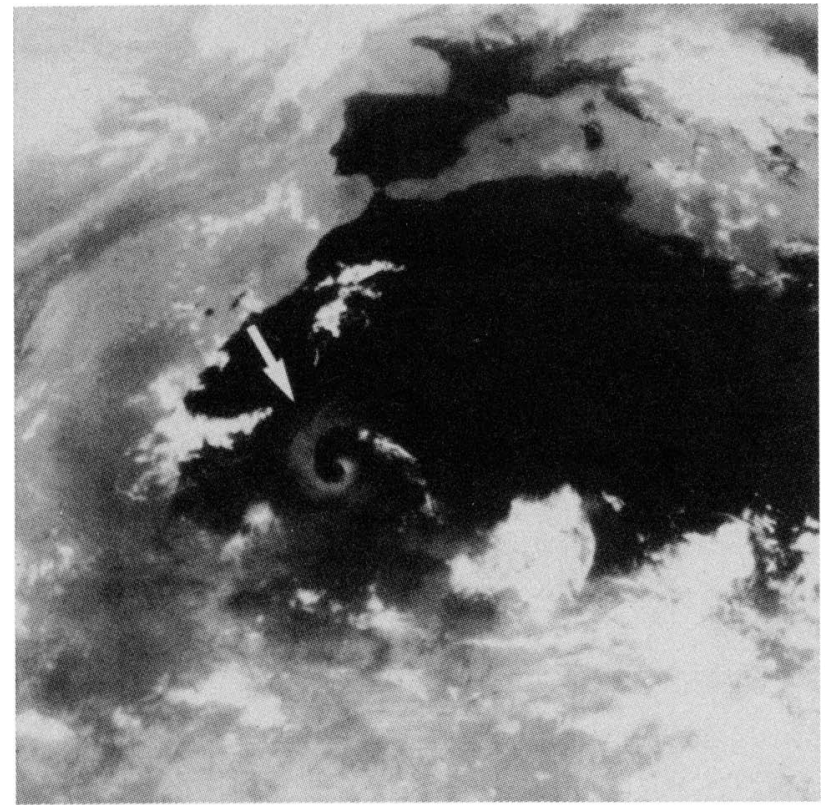

FIG. 6. Meteosat IR image of dust vortex (arrow) at 1155 UTC 29 August 1985. cases see Reed et al., 1986.

The accuracy of the initial state is crucially dependent upon the data assimilation system. It is possible to estimate the accuracy of the data assimilation system by partitioning the perceived forecast error into spatially correlated prediction errors and spatially uncorrelated observation errors (Hollingsworth and Lönnberg, 1986). The total forecast error, the difference between the model forecast and observations, consists of two parts-spatially correlated differences and spatially uncorrelated differences. The former are referred to as model-prediction error, and the latter as observation error. Figure 7 shows the total six-hour wind forecast error of the ECMWF model for the first quarter of 1984, together with the observational error and prediction error for the vector wind calculated for the North American and European radiosonde networks, respectively. The observational error is very similar in both areas with maximum values of around $5 \mathrm{~m} \cdot \mathrm{s}^{-1}$. The estimate of the radiosonde observational error is in agreement with that published by the World Meteorological Organization (WMO, 1983). Figure 8 shows the corresponding results for the height forecasts. The estimated prediction error for height is very similar over North America and Europe, which agrees with verification of the short-range forecasts against gridded analyses. The differences in the total forecast error in the two areas

\section{WIND / NORTH AMERICA}

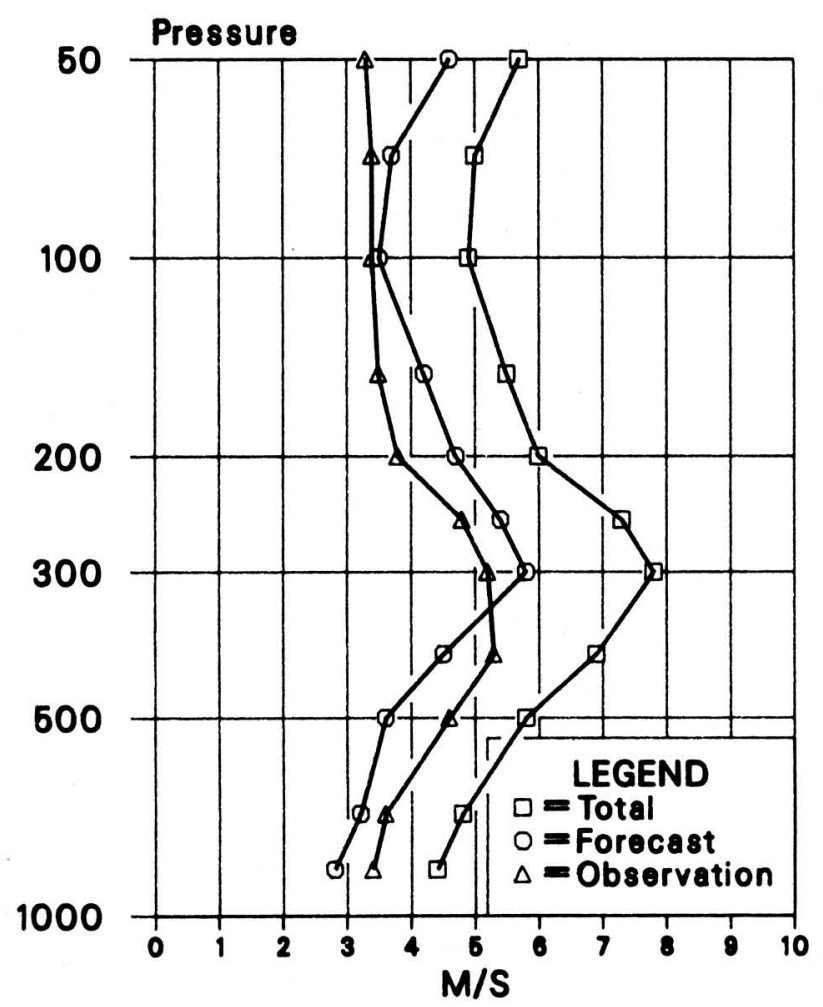

\section{WIND / EUROPE}

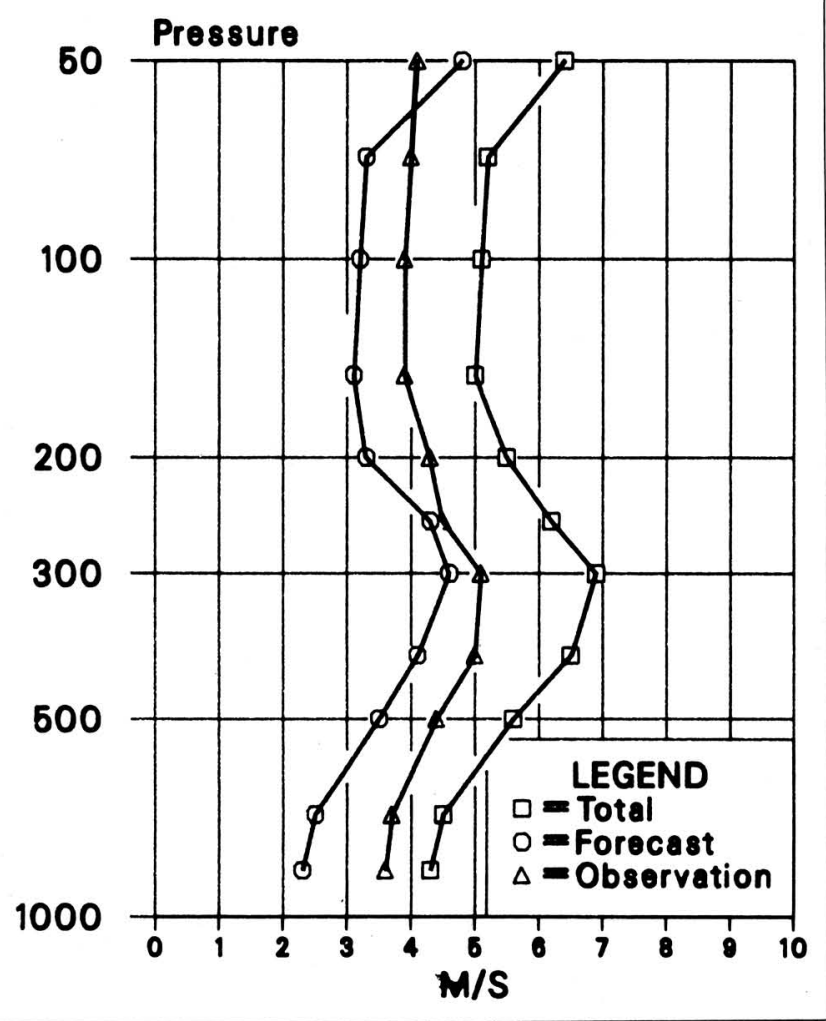

FIG. 7. The total 6-hour forecast error for the vector wind $\left(\mathrm{m}^{\cdot} \mathrm{s}^{-1}\right)$ using screened data for North America and Europe, and the calculated model prediction error and observational error as indicated in the legend. (Hollingsworth et al., 1986). 
arise from the higher level of observational error in the European network; the noise must arise from the fact that, contrary to the situation in North America many, different sonde types are used in Europe. The analysis system removes this noise, so that the verification against gridded analyses shows a lower level of error. It is interesting to note that the synoptic-scale six-hour prediction errors for height and wind over Europe and North America are comparable in magnitude to the observational error of individual radiosonde stations.

An interesting comparison of present-day data assimilation systems to those a decade ago can be seen in Fig. 9. Here the total forecast errors of short-range forecasts over North America are compared with those discussed by Hollett (1975) for similar forecasts for the same area and verified in the same way. Hollett used the Canadian five-level primitive-equation model, and his data pertain to the period August to October 1974. The ECMWF data are shown for the period January to March 1984 and June to August 1984 to indicate the seasonal variations. Assuming that there have been only modest changes in instrumental accuracy over the intervening time, it is clear that the accuracy of the forecasts must have improved substantially. Many factors may have contributed to this improvement, including higher model resolution and better analysis and initialization techniques.

\section{Comparison of the old and the new analyses for 1979}

The data from the Global Weather Experiment in 1979 were analyzed by the two authorized level IIIb data centers, the
Geophysical Fluid Dynamics Laboratory (GFDL) and ECMWF. This work, which required considerable resources in manpower and computer time, took place during 1980 and 1981 using the state-of-the-art data assimilation system as developed at the two institutions (Bengtsson et al., 1982; Ploshay et al., 1983; Stern et al., 1984). The analyzed data sets were made available to the scientific community via the World Data Centers. By now, a large number of diagnostic studies, prediction experiments, etc., have been carried out (see for example, NAS, 1985).

Although the observations that were used in preparing the analyses at GFDL and ECMWF were practically identical, significant differences were found between the two sets of analyses. The greatest differences were found in data-sparse regions such as the tropics; in these areas the analysis depends heavily upon the first guess from the model.

Since the production of the first FGGE data set (the Main FGGE Level III-b data set) in 1980, the ECMWF forecasting system has changed considerably (Tables 2 and 3; Uppala, 1987). Some of these changes have led to a more realistic analysis of the divergent component of the wind and hence significantly affected the calculation of second order diagnostic quantities (energy transformation and energy fluxes). For the sake of brevity, the Main ECMWF FGGE Level III-b data set will be referred to as the old analysis and the Final ECMWF FGGE Level III-b data set will be referred to as the new analysis. We present below a brief comparison of the old and the new analyses.

a. Vertical motion and velocity potential

Figure 10 shows the zonally averaged vertical motion for the

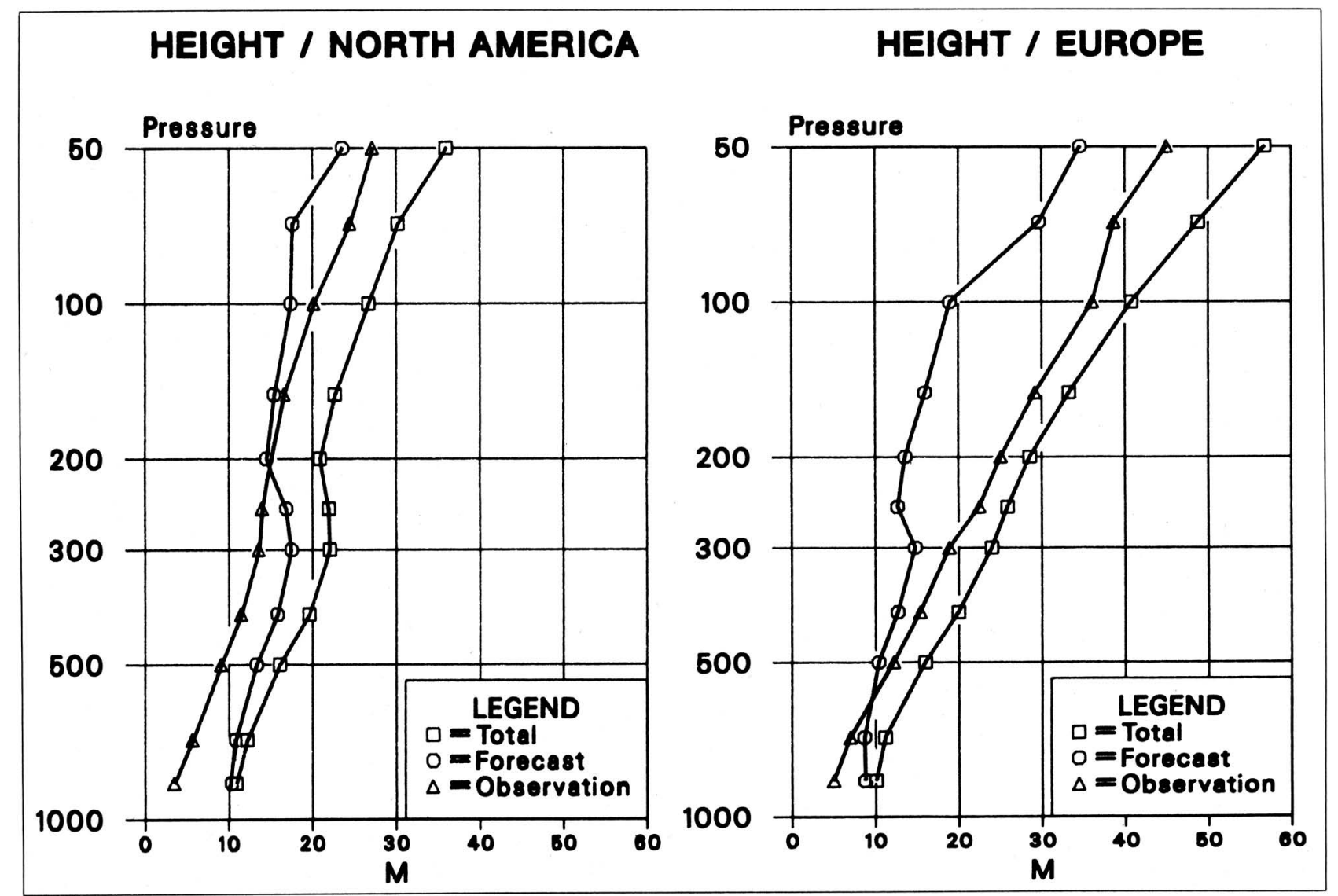

FIG. 8. As in Fig. 7 for the height in meters. (Hollingsworth et al., 1986). 


\section{HEICHT / NORTH AMERICA}

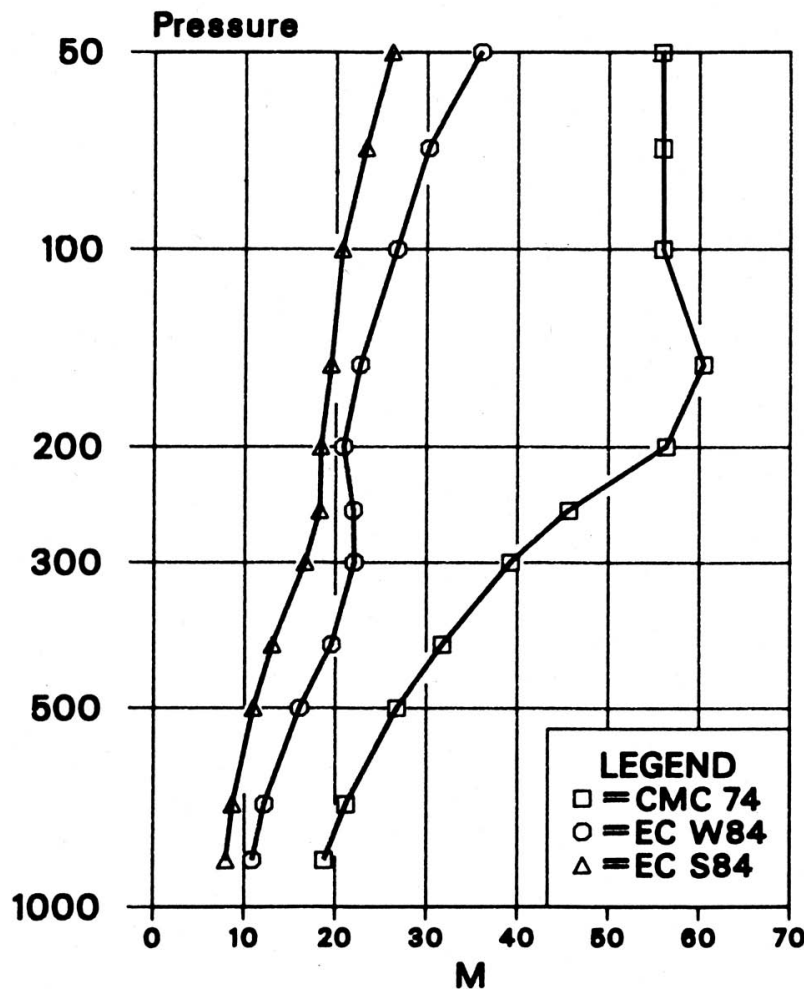

Fig. 9. The total six-hour forecast error for height (in meters), from verifications against North American radiosonde data. From Hollett (1975) indicated by CMC74, and for the ECMWF forecasts for January to March 1984 (indicated by EC W84) and June to August 1984 (indicated by EC S84). (Hollingsworth et al., 1986).

700 to $300-\mathrm{hPa}$ layer. The values are given as seasonal means from the winter 1981-1982 through and including the winter 1986-1987. Major changes in the forecasting system are indicated at the bottom of the figure. The enhancement of the meridional circulation can clearly be seen, in particular from the middle of 1985. Tiedtke et al. (1987) have shown that the changes in the physical parameterization, in particular the introduction of shallow convection and modification to the Kuo convection scheme, were crucial in creating a more intense Hadley circulation. Figure 11 shows the vertical-latitudinal cross section of zonally averaged vertical motion for old and new analyses. A deeper and more intense Hadley circulation is clearly seen in the new analyses.

Figure 12 shows the mean velocity potential at $200 \mathrm{mb}$ for the period 1-20 May 1979 from the final new analysis (top) and the old analysis (bottom). It is again clear that the Walker circulation is more intense in the new analyses. The general pattern of the velocity potential field remains very similar, but the magnitudes of the maxima and minima are significantly different.

In summary, improvements in the parameterizations of physical processes and improvements in data assimilation systems have enhanced the divergence and vertical motion fields and produced more intense Hadley and Walker circulations.

\section{b. Energetics}

Figure 13 gives the global energetics and energy conversions

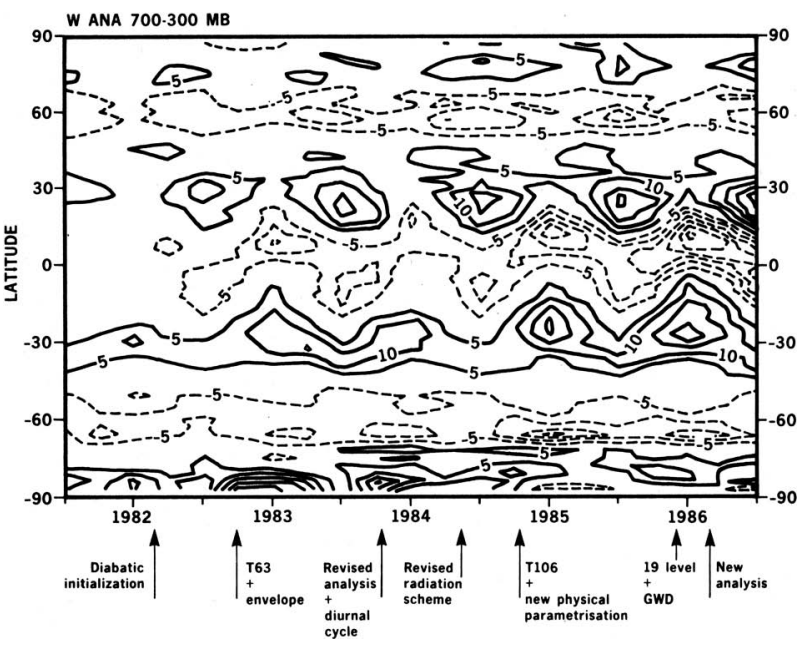

FIG. 10. Zonally averaged vertical motion $\left(10^{-3} \mathrm{~Pa} \cdot \mathrm{s}^{-1}\right)$ as analyzed by the ECMWF forecast model for the period 1 January 1982 to 31 December 1986. Important model changes are indicated at the bottom.

FEB.1979 EC-ANA
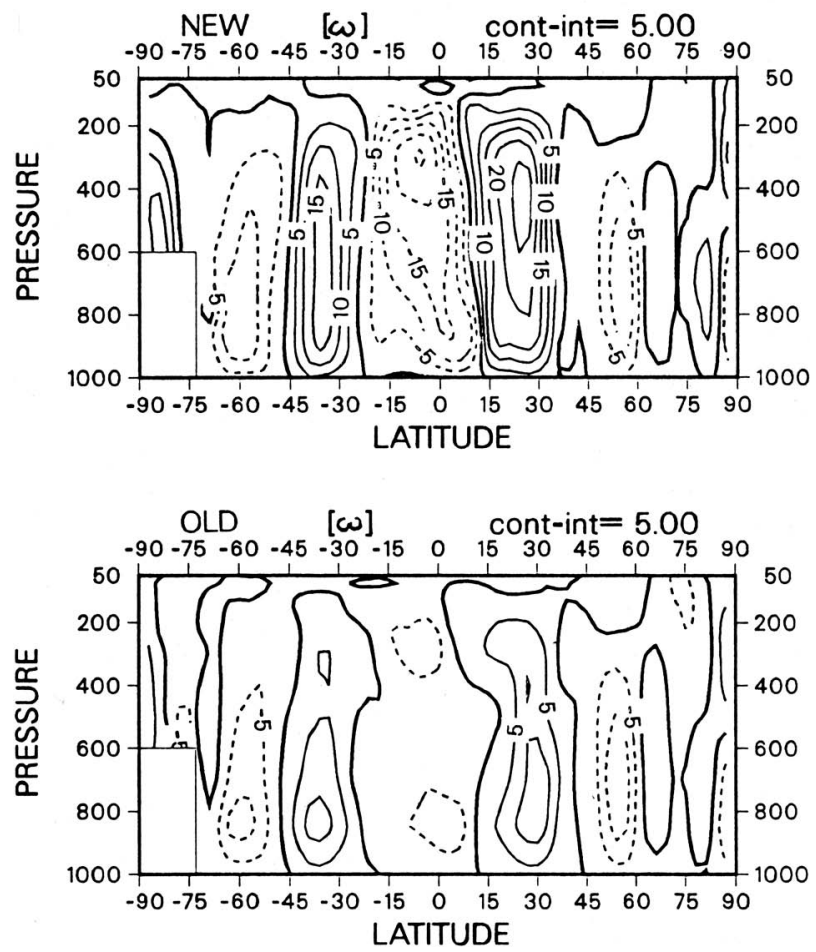

FIG. 11. Zonally averaged vertical motion $\left(10^{-3} \mathrm{~Pa} \cdot \mathrm{s}^{-1}\right)$ for new and old analyses for February 1979.

for new and old analyses for February 1979. It can be seen that the available potential and kinetic energies are higher in the new analysis and, in particular, the eddy available potential energy and the eddy kinetic energy are higher by more than 10 percent in the new analyses. Even bigger differences are seen in the generation, dissipation, and conversion terms. The generation of eddy-available potential energy is of opposite sign in the two analyses. Figure 14 shows a time series of eddyavailable and kinetic energy for February 1979. The time-fluc- 

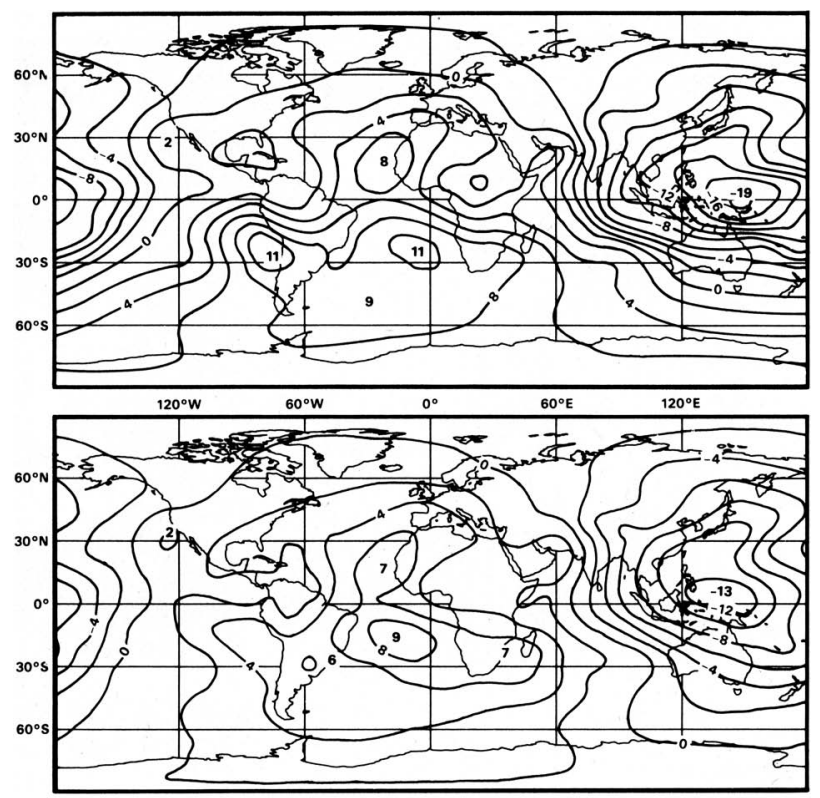

FIG. 12. Mean velocity potential at $200 \mathrm{mb}$ for the period 1-20 May 1979 for the new and old analyses. Units $2 \times 10^{-6} \mathrm{~m}^{2} \cdot \mathrm{s}^{-1}$. The divergent wind is directed from lower to higher values of the velocity potential and proportional to its gradient.

tuations in both cases are very similar; however, as noted in Fig. 13, the values of eddy-available and kinetic energies in the new analysis are higher by more than 10 percent compared to the old analysis.

\section{c. Heat flux and wind stress}

A detailed intercomparison was undertaken between the T63 and T106 model prior to the operational introduction of the T106 model on 1 May 1986. The difference in the physical parameterization is given in Table 2 . Figures 15 and 16 give the accumulated sensible heat flux for an ensemble of twenty 24-hour forecasts, 11 April 1985 to 30 April 1985. The two fields are not very different from one another except in the areas of intense sensible heat flux (e.g. the Gulf stream and the Kuroshio). There are, however, large differences between the latent heat flux in the old (Fig. 17) and the new (Fig. 18) models. In the central Pacific region, for example, the old latent heat flux is only half as large as the new values. In general, the old analysis severely underestimates the latent heat flux over the oceans. Introduction of shallow convection and a better boundary-layer formulation lead to a more realistic upward transport of moisture from the boundary layer, giving rise to a larger latent heat flux from the ocean. Corresponding surface stresses from the old model (Fig. 19) and the new model (Fig. 20) are quite similar for a 24-hour forecast starting from the same initial condition.

\section{Possible applications of an internally- consistent long-term multi-variate data set for the atmospheric circulation}

Atmospheric data sets produced by the operational NWP centers have been the primary source of data for a large number of diagnostic studies of the mean climate as well as the intrasea-

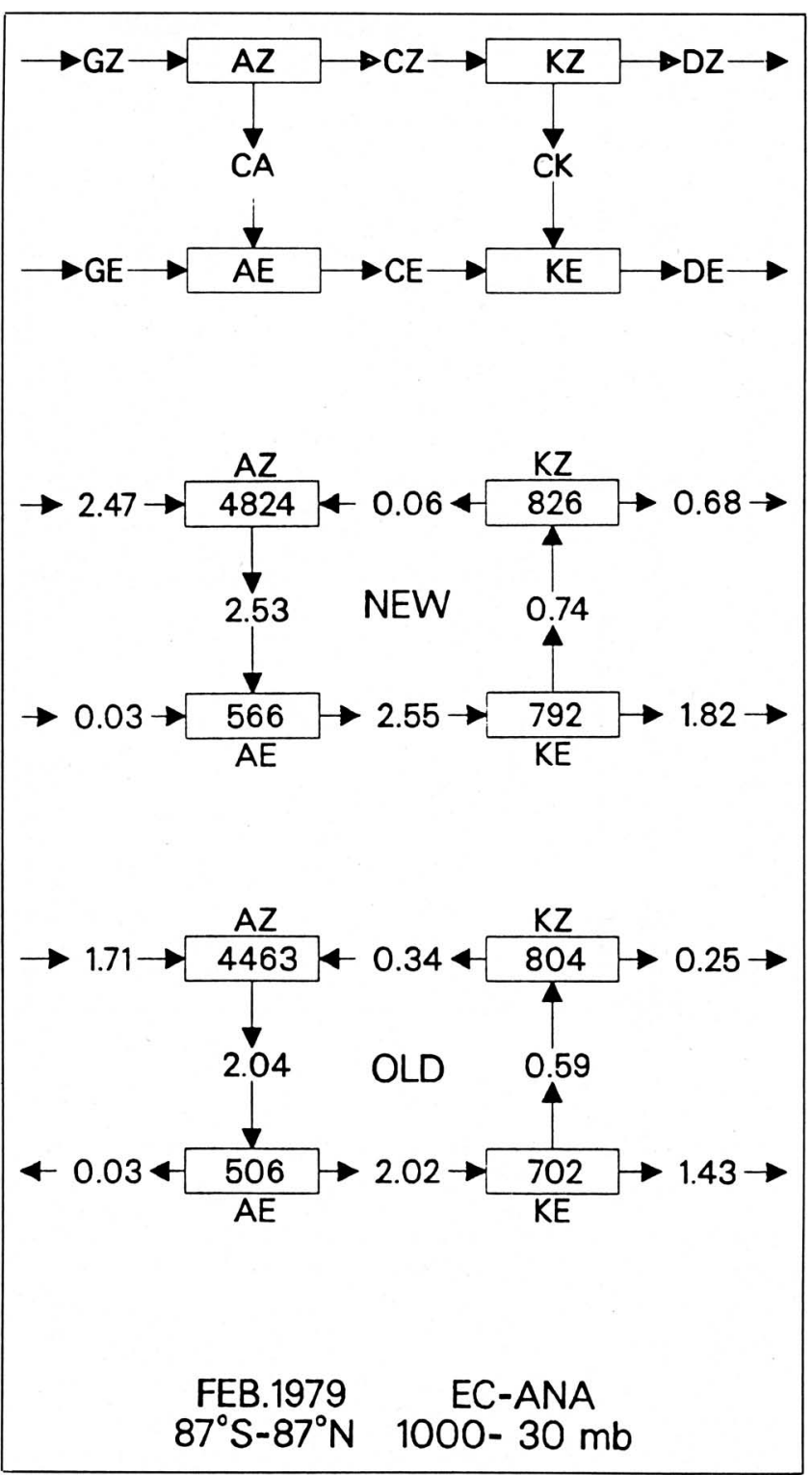

FIG. 13. Global energetics for the new and old analyses for February 1979.

sonal and interannual variability of the regional and global atmospheric circulation. Improvements in the analysis and forecast systems can produce qualitative and quantitative changes in the spatial and temporal variability of the atmospheric circulation. Due to frequent changes in the analysis and forecast system, it is also possible to introduce spurious interannual variability that may be difficult to distinguish from the natural variability of the atmospheric circulation (Sardeshmukh and Hoskins, 1987). An internally consistent data set can be quite useful in detecting changes in decadal mean climate, for example those suspected to be due to changes in $\mathrm{CO}_{2}$ and other trace gases.

Some of the modeling and diagnostic study areas that can be addressed with an internally homogeneous long-term data set for the atmospheric circulation are described below.

\section{a. Mean Climate}

A decadal or longer time-mean circulation for earth's atmo- 

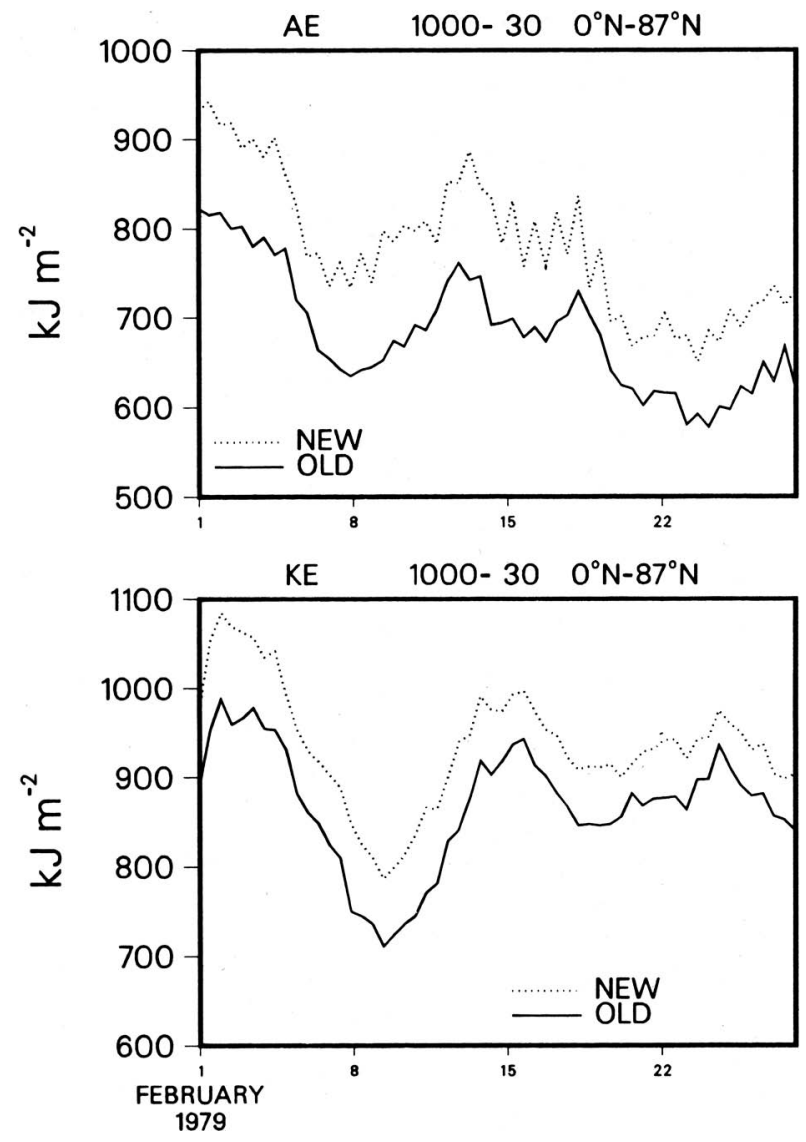

FIG. 14. Eddy-available and kinetic energy for February 1979 for new and old analyses.

sphere has never been calculated using a homogeneous data set. Reanalysis of data for a 20 -year period can provide descriptions of decadal means as well as changes from one decade to the other.

\section{b. Forcing for the ocean models}

It is essential to know the surface stress and surface-heat flux at the ocean surface in order to simulate the oceanic circulation and its interannual variability. Accurate specification of the atmospheric forcing is especially important in the context of TOGA and WOCE, because the interannual variability of tropical ocean circulation and sea-surface temperature (SST) is largely and directly determined by the atmospheric forcing. There does not yet exist an internally consistent and homogeneous data set for surface stress and surface heat fluxes that can be used to drive the tropical-ocean models. In order to determine the predictability of the tropical-ocean circulation and tropical SST, and to study the prospects for extended range forecasting using coupled models, it will be necessary to produce dynamically consistent long-term data sets of atmospheric forcing.

\section{c. Global hydrological cycle}

Due to the absence of rainfall measurements over the oceans, and uncertainties in the calculation of evaporation over both land and ocean, it has not been possible to make a quantitative determination of each of the components of the global hydrological cycle. A consistent data set for the atmospheric cir-

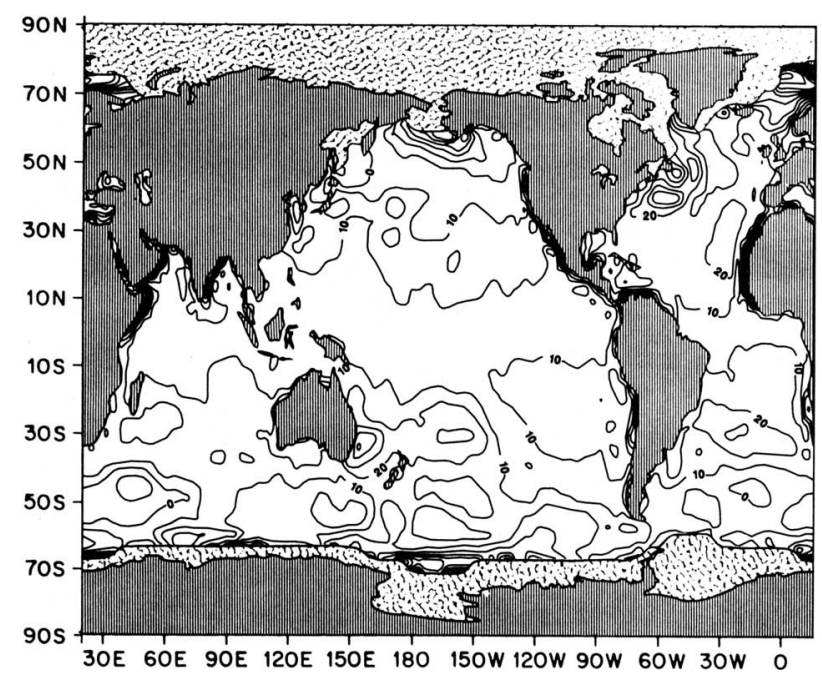

FIg. 15. Sensible heat flux $\left(\mathrm{W} \cdot \mathrm{m}^{-2}\right)$ accumulated over 24-hour forecasts for the T63 model with old physics. Ensemble of twenty 24hour forecasts (11 April to 30 April 1985).

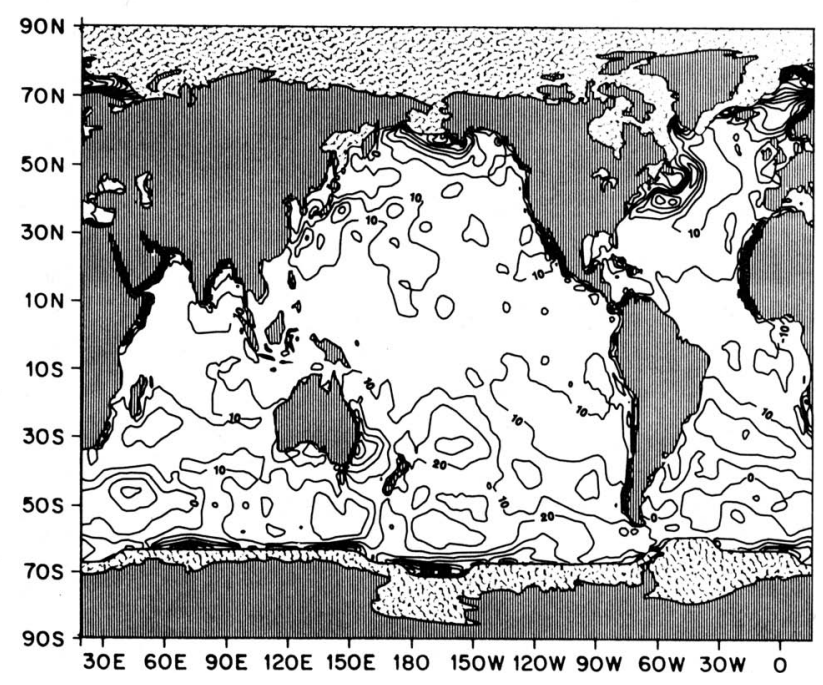

FIG. 16. Same as Fig. 15, for the T106 model with new physics.

culation and moisture can be used for calculating the vertically integrated moisture flux convergence which can be combined with the available, albeit uncertain, data sets of evaporation and precipitation over land to define the complete hydrological cycle. However, for such an indirectly calculated field as precipitation to be meaningful, the global divergence field must be reasonably accurate.

The fact that the atmospheric models have improved to the extent that they produce reasonable forecasts of development and propagation of synoptic-scale circulation features even over the oceans, including rapid developments strongly influenced by the release of latent heat, it is reasonable to conjecture that the diabatic forcing is being calculated reasonably well, and therefore one can further conjecture that the rainfall distribution over oceans may not be too unreasonable either.

\section{d. Atmospheric energetics}

As was shown in Figs. 13 and 14, reanalysis of FGGE data gave rise to significant changes in atmospheric circulation vari- 


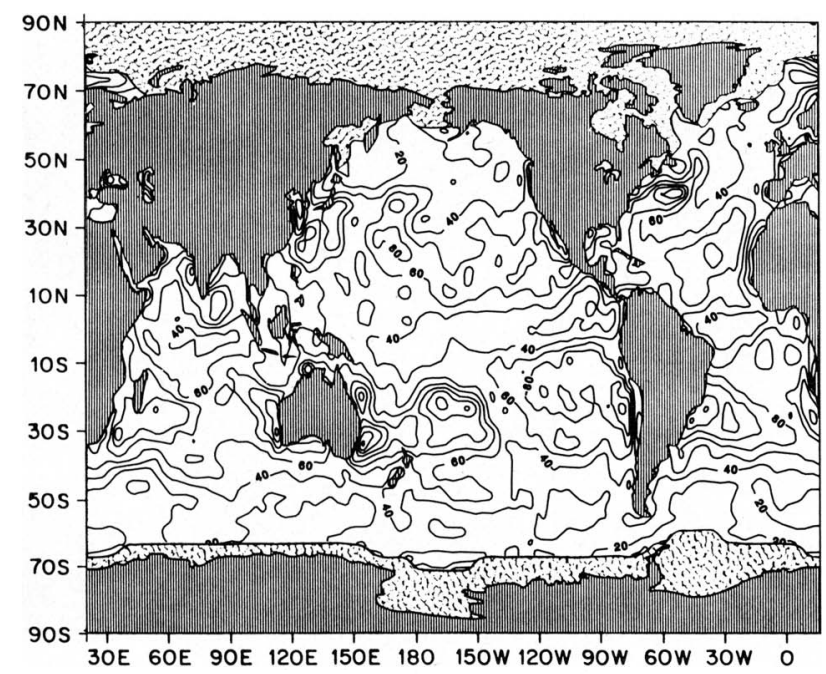

FIG. 17. Latent-heat flux $\left(\mathrm{W} \cdot \mathrm{m}^{-2}\right)$ accumulated over 24-hour forecasts for the T63 model with old physics. Ensemble of twenty 24-hour forecasts (11 April to 30 April 1985).

ables. The impact upon the derived quantities, and particularly quadratic quantities such as momentum, energy, and moisture transports are substantial. It would be particularly desirable to recalculate the atmospheric energetics using a consistent data set over a long period.

\section{e. Structure and variability of vertical velocity, divergence, and diabatic heating}

In the past it has been particularly difficult to produce reliable fields of non-measurable quantities such as vertical velocity, divergence, and diabatic heating. These are needed particularly for carrying out comprehensive and complete budget studies for the atmospheric circulation.

\section{f. Intraseasonal variability}

Global observations of vertical velocity, heating, and divergence would be extremely useful for diagnosing the mechanisms of intraseasonal variability. Typical examples are blocking phenomena in mid-latitudes, and 30 to 60 day oscillations in the tropics. Homogeneous and internally consistent data sets can be used to gain insight into the processes responsible for the onset, growth, and persistence of such phenomena.

\section{g. Land surface processes}

Due to the lack of adequately high-resolution and high-frequency (sufficient to resolve the diurnal variation) observations of rainfall, sensible-heat flux, evapotranspiration, and the radiation budget at the surface, it has not been possible to study land-surface processes adequately. Reanalysis with a high-resolution atmospheric model and a realistic biosphere model can provide a long-time series for these data sets.

\section{Concluding remarks and suggestions}

The four-dimensional data assimilation system with a realistic physical model, of the type routinely employed in operational NWP, offers the most comprehensive technique for integrating

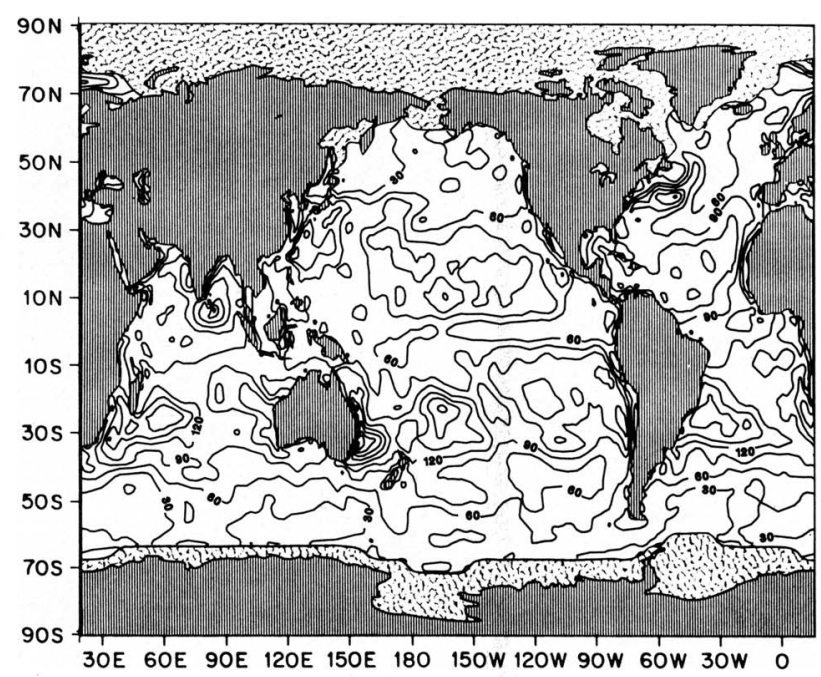

FIG. 18. Same as Fig. 17, for the latent heat flux $\left(\mathrm{W} \cdot \mathrm{m}^{-2}\right)$ for the T106 model with new physics.

space and in situ observations to produce internally consistent, homogeneous, multivariate data sets for the earth's climate system. Such data sets are required for the success of the emerging World Climate Research Program, the International Geosphere-Biosphere program, and projects conducted under the auspices of global change and earth-system science.

The existing model-based data sets produced in the course of operational NWP suffer from various limitations due to frequent changes in the forecast assimilation systems. The analyses also do not include delayed mode data because of data cut-off constraints in operational NWP. Due to deficiences and limitations in the Global Telecommunications System (GTS), up to 30 percent of available meteorological bulletins (surface, TEMP, PILOT) do not reach the forecasting centers and are consequently not made available to the numerical forecasting centers (ECMWF, 1987). However, it is hardly a practical proposition to collect all this delayed observation for a longer period, but special efforts could be envisaged to obtain additional observations, such as ship observations, in data-sparse regions.

Even if no additional data sets were available it would be worthwhile to carry out a reanalysis of the past data sets using an improved forecast and assimilation system for the entire period. This would enable us to study climate change with homogeneous data sets. Reanalysis will be of special importance in the future because quite large increments in the satellite-derived data sets are expected to occur in the coming decades.

The current data assimilation systems, particularly satellite retrieval techniques, have been designed and developed under the constraints of operational NWP. A massive reanalysis effort will encourage and facilitate major research projects on satellite data retrievals and assimilation systems, such as self adjoint techniques which can use future data sets.

A model-based reanalyzed data set will have the benefit of utilizing the most accurate description of the boundary conditions at the earth's surface during the forecast and assimilation cycle.

Although this paper presents illustrations only for the global 


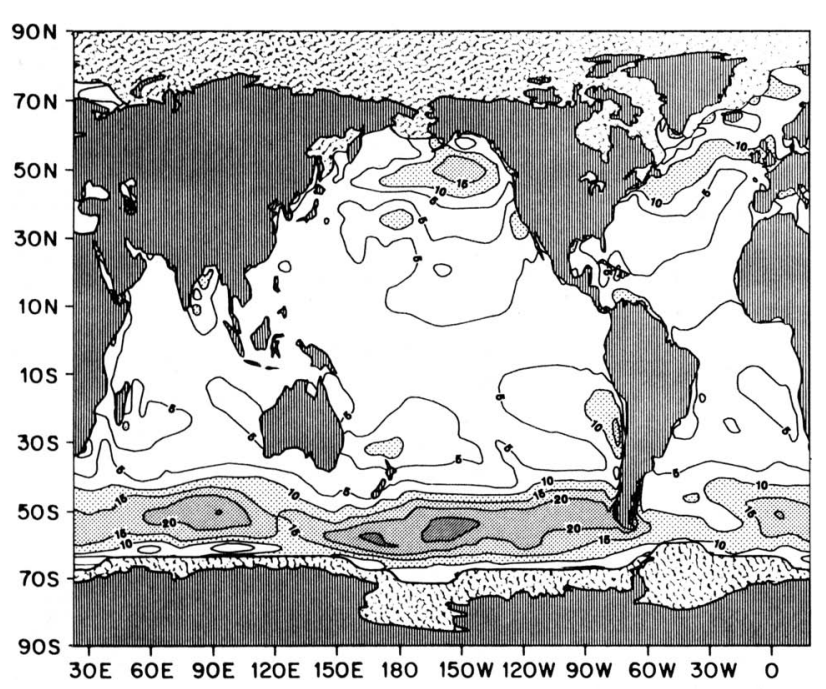

FIG. 19. Surface stress $\left(10^{-2} \mathrm{~N} \cdot \mathrm{m}^{-2}\right)$ accumulated over 24-hour forecasts for the T63 model with old physics. Ensemble of twenty 24hour forecasts (11 April to 30 April 1985).

atmospheric models, the technique is equally applicable for the analysis of the global ocean circulation and mesoscale weather systems.

Reanalysis of atmospheric data sets with the observed ocean temperature will produce wind stress and heat flux at the ocean surface that in turn will serve as the forcing functions for the ocean models employed for an oceanic forecast and assimilation system. Likewise, a mesoscale model can be utilized to assimilate all the available observations in a region to produce a comprehensive description of the four-dimensional structure of mesoscale systems.

Current and future observing systems are very expensive and dominate the expenditure budget of the Meteorological Services. It would be desirable to extract the maximum information possible from the routine observations taken in situ or from a satellite-based observing system. Although a massive reanalysis project would appear to require large computer resources, the cost involved would be modest in comparison to the cost of the observing systems. The development of archiving systems by making use of compact disc (CD-ROM) offers great possibilities for the scientists in getting access to huge data bases in an affordable way. This has recently been demonstrated by Mass et al. (1987).

In order to have available the best possible data base, arrangements should be made to archive the data sets received after the cut-off time imposed by operational NWP. The collection, processing, and archiving of all the in situ and spacebased observations should be carried out in such a way that a reanalysis can be performed at a later date.

Additional research should also be encouraged to develop satellite retrieval techniques, and to develop suitable models for the treatment of atmosphere-ocean and atmosphere-land interactions and assimilation of conventional and satellite data sets in the boundary layers of both the atmosphere and the oceans.

One of the immediate problems is to develop suitable techniques to evaluate the relative merits of the old and new analyses. One possible way to address this question is by evaluation

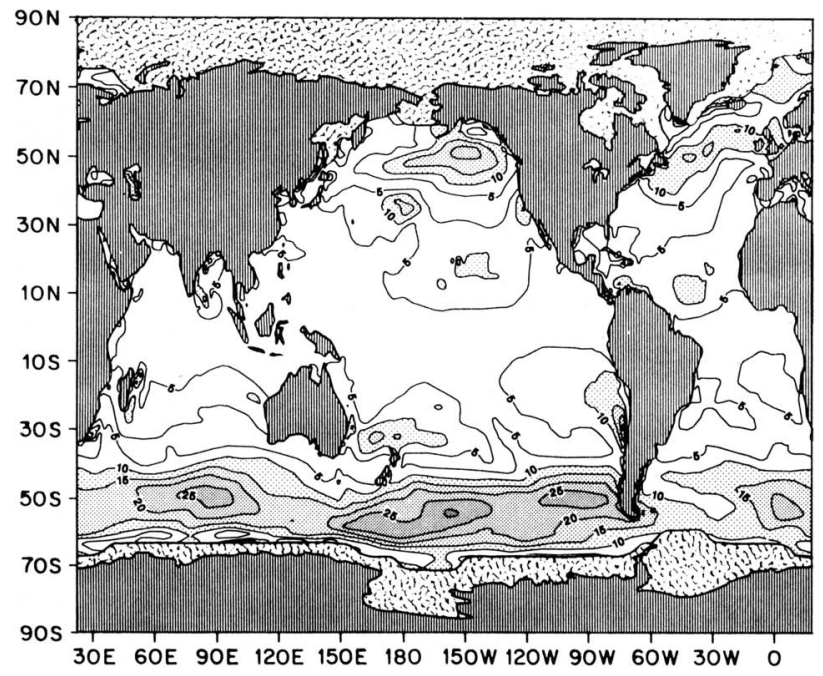

FIG. 20. Same as Fig. 19 for surface stress $\left(10^{-2} \mathrm{~N} \cdot \mathrm{m}^{-2}\right)$ for the T106 model with new physics.

of momentum and vorticity balances (Sardeshmukh and Hoskins, 1987), which should be relatively less sensitive to the parameterization of diabatic processes.

There is no doubt that a reanalysis of global data over, say, a period of ten years is a considerable effort, both in manpower and computer resources. Furthermore, as the data assimilation system will continue to evolve, we will in principle always have a situation where one could argue that previous data sets should be reanalyzed with the best available system. However, it appears reasonable from a practical point of view to select a time period when available data assimilation systems have reached an apparent plateau in their evolution. We believe that could happen within one or two years, when present techniques based on optimum interpolation and normal mode initialization would have come to a mature stage. New techniques based on four-dimensional variational techniques and adjoint models will probably not reach a mature state before 1995. For this reason we suggest that a reanalysis project be initiated to reanalyze a ten-year period 1979 to 1988 using state-of-the-art data assimilation systems including a model with the highest possible resolution. The project should commence in 1989 or 1990 with the objective to produce a full data set within a period of about two years.

Acknowledgments. The authors would like to thank J. L. Kinter for his constructive comments on an earlier version of the paper. The authors greatly acknowledge the assistance of staff members from ECMWF and COLA, in particular W. Heckley, K. Arpe, and H. Böttger. We thank Marlene Schlichtig (COLA) and Astrid Dinshawe (ECMWF) for typing the manuscript and preparing the figures.

This research was supported by NSF Grant ATM-8414660 and NASA Grant NAGW-1166.

\section{Appendix 1: a measure of skill}

Monthly means of daily hemispheric averages of the anomaly correlations $(A C)$ and the standard deviation $(F S T D)$ of forecast errors are used to define forecast skill, $G$ in days, according 
to the following calculation: (i) a mean score, $\bar{S}$, is formed by averaging the following four scores $S_{1}=1-A C(Z), S_{2}=$ $(F S T D(Z) / P S T D(Z))^{2}, S_{3}=1-A C(T), S_{4}=(F S T D(T) /$ $\operatorname{PSTD}(T))^{2}$;

$$
\bar{S}=.25\left(S_{1}+S_{2}+S_{3}+S_{4}\right),
$$

PSTD is the standard deviation of the error of a persistence forecast. $T$ refers to temperature and $Z$ refers to height. The scores are calculated as averages for the region between $20^{\circ} \mathrm{N}$ and $82.5^{\circ} \mathrm{N}$; from 1000 to $200 \mathrm{mb}$ for $Z$, and from 850 to 200 mb for $T$. (ii) Values of $\bar{S}$ are obtained for each of the first 7 forecast days: $\bar{S}_{\mathrm{j}} \mathrm{j}=1,7$. A straight line is fitted to these points, $\hat{S}=\hat{a} x$ where $x$ is the forecast time in days and

$$
\hat{a}=\frac{1}{7} \sum_{\mathrm{j}=1}^{7} \frac{\bar{S}_{\mathrm{j}}}{x_{\mathrm{j}}} \quad S_{\mathrm{j}}=1,7 \text { days }
$$

$\bar{S}=0.5$ is taken as the limit of skillful forecast and the corresponding limit of predictability $x=G$ in days is given by

$$
G=0.5 / \hat{a}(G \text { is plotted in Figure 1.) }
$$

\section{References}

Anthes, R. A., 1972: Development of asymmetrics in a three-dimensional numerical model of the tropical cyclone. Mon. Wea. Rev., 100, 461-476.

Arpe, K. A. Hollingsworth, A. C. Lorenc, M. S. Tracton, S. Uppala and P. Kăllberg, 1985: The response of numerical weather prediction systems to FGGE II-b data. Part II: Forecast verifications and implications for predictability. Quart. J. Roy. Meteor. Soc., 111, 67102.

Bengtsson, L., 1985: Medium range forecasting at the ECMWF. Advances in Geophysics, 28B, 3-56.

Bengtsson, L., M. Kanamitsu, P. Kăllberg and S. Uppala, 1982: FGGE 4-dimensional data assimilation at ECMWF. Bull. Amer. Meteor. Soc., 63, 29-43.

Dell'Osso, L. and L. Bengtsson, 1985: Prediction of a typhoon using a fine-mesh NWP model. Tellus, 37A, 97-105.

ECMWF, 1987: Planning meeting on data for global models. Final report 4-6 March 1987, available from ECMWF.

Hollett, S. R., 1975: 3-dimensional spatial correlations of PE forecast errors. M.Sc. Thesis. Department of Meteorology, McGill University, 73 pp.

Hollingsworth, A., U. Cubasch, S. Tibaldi, C. Brankovic, T. N. Palmer and L. Campbell, 1987: "Mid-latitude atmospheric prediction on time scales of 10-30 days." In Atmospheric and Oceanic Variability. Ed.: H. Cattle, Roy. Meteor. Soc., pp. 117-152.

Hollingsworth, A. and P. Lönnberg, 1986: The statistical structure of short range forecast errors as determined from radiosonde data. Part I: The wind field. Tellus, 38A, 111-136.
Hollingsworth, A., A. C. Lorenc, M. S. Tracton, K. Arpe, C. G. Cats, S. Uppala and P. Kăllberg, 1985: The response of numerical weather prediction systems to FGGE II-b data. Part I: Analyses. Quart. J. Roy. Meteor. Soc., 111, 1-66.

Hollingsworth, A., D. B. Shaw, P. Lönnberg, L. Illari, K. Arpe and A. J. Simmons, 1986: Monitoring of observations and analysis quality by a data assimilation system. Mon. Wea. Rev., 114, 861-879.

Lange, A. and E. Hellsten, 1983: Results of the WMO/CAS NWP data study and intercomparison project for forecasts for the Northern Hemisphere in 1981-82. WMO Short- and Medium-Range Weather Prediction Research, Publication Series No. 2, WMO, Geneva.

Lorenz, E. N., 1982: Atmospheric predictability experiments with a large numerical model. Tellus, 34, 505-513.

Mass, C. F., H. F. Edmon, H. J. Friedman, N. R. Cheney and E. E. Recker, 1987: The use of compact discs for the storage of large meteorological and oceanographic data sets. Bull. Amer. Met. Soc., 68, 1556-1557.

NAS, 1985: Proceedings of the First National Workshop on the Global Weather Experiment. Vol. I and II. National Academy Press, Washington, D.C.

Ploshay, J. J., R. White and K. Miyakoda, 1983: FGGE III-b daily global analyses. Part I. NOAA Data Report, ERL GFDL-1.

Reed, R. J., A. Hollingsworth, W. A. Heckley and F. Delsol, 1986: An evaluation of the ECMWF operational forecasting system in analyzing and forecasting tropical easterly wave disturbances. Part 1: Synoptic investigations. ECMWF Tech. report No. 58, pp. 1-75.

Sardeshmukh, P. and B. J. Hoskins, 1987: On the deviation of the divergent flow from the rotational flow: The $\chi$ problem. $Q . J . R$. Meteorol. Socl., 113, 339-360.

Simonot, J. Y. and H. LeTreut, 1987: Surface heat fluxes from a numerical weather prediction system. Climate Dynamics, 2, 11-28.

Smagorinsky, J., K. Miyakoda and R. F. Strickler, 1970: The relative importance of variables in initial conditions for dynamical weather prediction. Tellus, 22, 141-157.

Stern, W. R., J. J. Ploshay and K. Miyakoda, 1984: Continuous data assimilation at GFDL during FGGE. ECMWF Seminar Proc. Data Assimilation Systems and Observing System Experiment with Particular Emphasis on FGGE, 125-156.

Tiedtke, M., W. A. Heckley and J. Slingo, 1987: Tropical forecasting at ECMWF: On the influence of physical parameterization on the mean structure of forecasts and analyses. Submitted to QJRMS.

Trenberth, K. E. and J. G. Olson, 1988a: Evaluation of NMC Global Analyses: 1979-1987. NCAR Technical Note, NCAR/TN$299+$ STR, Boulder, CO.

Trenberth, K. E. and J. G. Olson, 1988b: Intercomparison of NMC and ECMWF Global Analyses: 1980-1986. NCAR Technical Note, NCAR/TN-299+STR.

Tuleya, R., 1987: A numerical study of the genesis of tropical storms observed during the FGGE year. Mon. Wea. Rev., 116, 1188-1208.

Uppala, S., 1987: ECMWF Final Level IIIb Analysis: Analysis systems performance and impact on forecasts. To be published by the U.S. National Research Council.

WMO, 1983: Guide to meteorological instruments and methods of observations. 5th Edition. WMO, Case Postale No. 5, CH1211, Geneva 20, Switzerland. 